\title{
Effects of Raman scattering in quantum state-preserving frequency conversion
}

Friis, Søren Michael Mørk; Andersen, Lasse Mejling; Castaneda, Mario A. Usuga; Pedersen, Anders Tegtmeier; McKinstrie, Colin J.; Rottwitt, Karsten

Published in:

Proceedings of 2014 Conference on Lasers and Electro-Optics (CLEO)

Publication date:

2014

Document Version

Publisher's PDF, also known as Version of record

Link back to DTU Orbit

Citation (APA):

Friis, S. M. M., Andersen, L. M., Castaneda, M. A. U., Pedersen, A. T., McKinstrie, C. J., \& Rottwitt, K. (2014). Effects of Raman scattering in quantum state-preserving frequency conversion. In Proceedings of 2014 Conference on Lasers and Electro-Optics (CLEO) IEEE.

\section{General rights}

Copyright and moral rights for the publications made accessible in the public portal are retained by the authors and/or other copyright owners and it is a condition of accessing publications that users recognise and abide by the legal requirements associated with these rights.

- Users may download and print one copy of any publication from the public portal for the purpose of private study or research.

- You may not further distribute the material or use it for any profit-making activity or commercial gain

- You may freely distribute the URL identifying the publication in the public portal 


\title{
Effects of Raman scattering in quantum state-preserving frequency conversion
}

\author{
S. M. M. Friis, ${ }^{1}$ L. Mejling, ${ }^{1}$ M. A. Usuga, ${ }^{1}$ A. T. Pedersen, ${ }^{1}$ \\ C. J. McKinstrie, ${ }^{2}$ and K. Rottwitt ${ }^{1}$ \\ ${ }^{1}$ DTU Fotonik, Technical University of Denmark, Oersteds Plads 343, 2800 Lyngby, Denmark \\ ${ }^{2}$ Bell Laboratories, Alcatel-Lucent, Holmdel, New Jersey, 07733, USA \\ smmf@fotonik.dtu.dk
}

\begin{abstract}
We analyse frequency conversion by Bragg scattering numerically including Raman scattering. The frequency configuration that performs the best under influence of Raman noise results in $95 \%$ conversion over a $3.25 \mathrm{THz}$ bandwidth with a 2.5 -dB noise figure.

(C) 2014 Optical Society of America

OCIS codes: 190.4380 Nonlinear optics, four-wave mixing; 270.2500 Fluctuations, relaxations, and noise.
\end{abstract}

A crucial feature of quantum communication networks is the ability to alter the temporal mode profile as well as the frequency of a quantum state, while preserving other quantum properties [1]. It has been shown that the third-order effect in optical fibers enables quantum frequency conversion (QFC) [2]. In this case, one uses two strong pumps $\mathrm{p}$ and $\mathrm{q}$ that interact with a signal $\mathrm{s}$ and an idler $\mathrm{i}$. They fulfill the energy conservation $\omega_{\mathrm{p}}+\omega_{\mathrm{s}}=\omega_{\mathrm{q}}+\omega_{\mathrm{i}}$, and this specific setup is known as Bragg scattering (BS). BS has been studied extensively both for classical [3] and for quantum signals $[4,5]$. Using the third-order nonlinearity of silica fibers, BS also allows for QFC inside the low-loss communication window, which we focus on in this paper.

Higher order dispersion (HOD) and Raman scattering (RS) are known to severely limit the efficiency and quality of BS, but current analytical quantum approaches do not describe these effects. In [6], we presented a semi-classical method for predicting the quantum noise properties of parametric processes: an ensemble of $10^{5}$ fields are propagated through a fiber using four coupled equations that describe four-wave mixing. Terms accounting for stimulated RS and loss are also included. The ensemble resembles a coherent state, and in every numerical step, further fluctuations are added to account for spontaneous RS and coupling to the vacuum state. This method allows us to evaluate the conversion efficiency (CE) and the noise figure (NF) of the BS process including HOD, RS and loss. In this work, we analyse frequency conversion of classical continuous wave signals that are situated on both the Stokes (S) and anti-Stokes (AS) sides of the two pumps, which are driving the conversion.

Figure 1(a) shows four frequency configurations of BS, which are performing down conversion (DC) from the signal to the idler, where $\omega_{0}$ is the zero-dispersion frequency. By changing $\omega_{\mathrm{s}} \leftrightarrow \omega_{\mathrm{i}}$ and $\omega_{\mathrm{p}} \leftrightarrow \omega_{\mathrm{q}}$, up conversion (UC) is achieved. Diagrams (i)-(ii) are denoted nearby BS, and (iii)-(iv) distant BS; in (i) and (iii), the signal and idler are in the $\mathrm{S}$ configuration, and in (ii) and (iv), they are in the AS configuration. This distinction is important due to the asymmetry of Raman scattering. Note that the conversion span in frequency is $\delta$ in (i)-(ii), but it is $\delta+2 \Delta$ in (iii)-(iv), see Fig. 1(a). Figure 1(b) shows the Raman gain coefficient of silica (black), $g_{\mathrm{R}}\left(\Omega_{i j}\right)$, taken from Ref. [7]; the phonon equilibrium

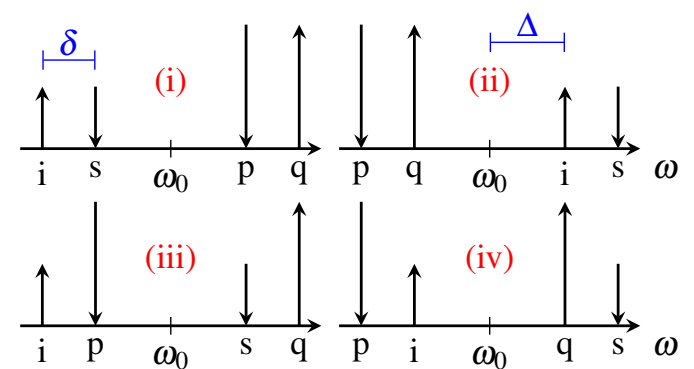

(a)

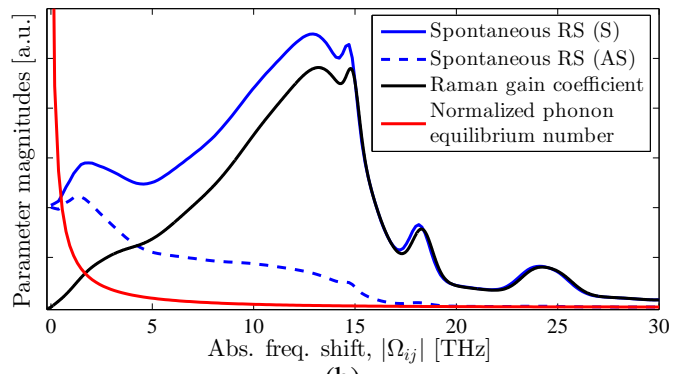

(b)

Fig. 1: (a) Diagrams (i)-(iv) show four configurations of BS performing DC from signal til idler; $\delta$ is the distance between side bands, $\Delta$ is the distance between the zero-dispersion frequency and the closest wave component. (b) Raman gain coefficient, phonon equilibrium number, and spontaneous RS rates spectra of silica. 


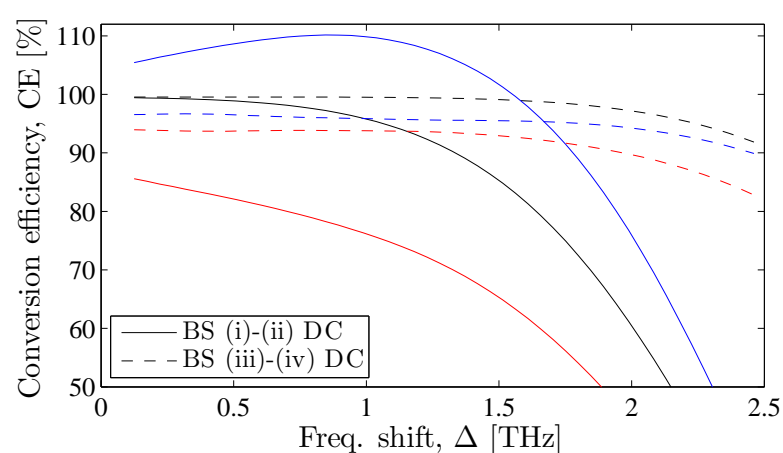

(a)

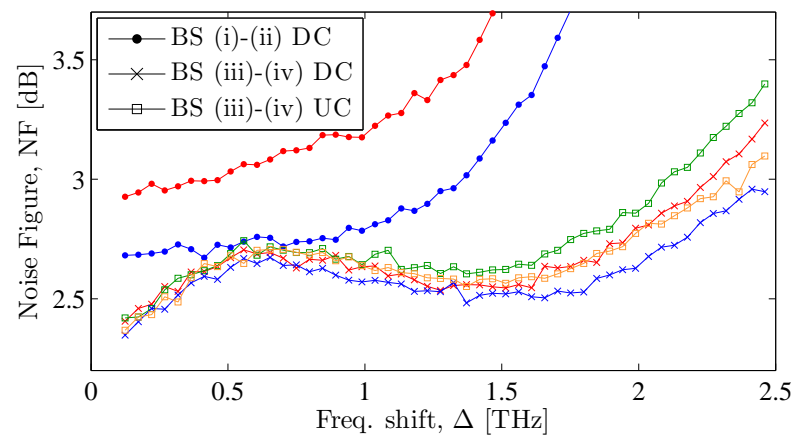

(b)

Fig. 2: (a) CE with RS and loss vs. $\Delta$ for DC for the $\mathrm{S}$ side (i)+(iii) (blue) and the AS side (ii)+(iv) (red), and without RS and loss (black). (b) NF for the same conditions inlcuding UC for the S side (orange) and the AS side (green).

number $n_{T}^{i j}=\left[\exp \left(\hbar\left|\Omega_{i j}\right| / k_{B} T\right)-1\right]^{-1}$ (red), where $\Omega_{i j}=\omega_{i}-\omega_{j}, \hbar$ is Planck's constant, $k_{B}$ is Boltzmann's constant, and $T$ is the temperature; and the $\mathrm{S}$ and AS spontaneous RS rates (blue). The latter are calculated as $R_{\mathrm{S}}=\left(n_{T}^{i j}+1\right) g_{\mathrm{R}}^{i j}$ and $R_{\mathrm{AS}}=n_{T}^{i j} g_{\mathrm{R}}^{i j}$ [8]. From Fig. 1(b), we see that spontaneous RS is more likely to occur on the $\mathrm{S}$ side (solid) than the AS side (dashed). Secondly, we observe two regions on the S side of lower spontaneous RS rate: at $\left|\Omega_{i j}\right|<1 \mathrm{THz}$ and near $\left|\Omega_{i j}\right|=5 \mathrm{THz}$.

Figure 2(a) shows the CE for each BS configuration in a $L=520 \mathrm{~m}$ fiber with a loss coefficient $\alpha=0.4 \mathrm{~dB} / \mathrm{km}$, fourth-order dispersion $\beta_{4}=-2.5 \times 10^{-55} \mathrm{~s}^{4} / \mathrm{m}$, and $T=300 \mathrm{~K}$; all other parameters are taken from [5]. Uneven dispersion terms cancel due to the symmetry of BS. For (i)-(ii) and (iii)-(iv), $\delta=1.48 \mathrm{THz}$ and $\delta=0.25 \mathrm{THz}$, respectively. HOD causes poor phase-matching for increasing $\Delta$, hence the decreasing efficiency. In (i)-(ii), stimulated RS causes the efficiency to increase at the S side (blue) and decrease at the AS side (red); in (iii)-(iv), stimulated RS decreases the CE slightly for both configurations. All UC simulations were indistinguishable from their corresponding DC curves and are therefore not shown.

Figure 2(b) shows the idler $\mathrm{NF}=\mathrm{SNR}_{\text {signal }}^{\text {in }} / \mathrm{SNR}_{\text {idler }}^{\text {out }}$ for all BS configurations; the UC curves for (iii)-(iv) are shown for the $\mathrm{S}$ side (orange) and the AS side (green). The two curves of (i)-(ii) represent a compromise between Raman gain and Raman noise: on the S side (blue), the idler receives a large amount of energy from the pumps, but also much noise. On the AS side (red), the idler receives only a little noise, but gives energy to the pumps. The compromise favours the (i) configuration. The NFs of (iii)-(iv) are all lower than the (i)-(ii) curves. Common for all of them is that the idlers are closer to one pump but not any further from the other; hence, the combined effect of RS is smaller. The evolution of the four curves may be understood from Figs. 1(b) and 2(a): the NF is increased in line with the curves of spontaneous RS and with decreasing CE due to HOD and the consequent phase-mismatch. Therefore, observing the region of low spontaneous RS around $\left|\Omega_{i j}\right|=5 \mathrm{THz}$, the low NF in (iii)-(iv) near $\Delta=1.5 \mathrm{THz}$ is due to the combined effects of spontaneous RS and dispersion, which increases the NF from $\Delta<1.5 \mathrm{THz}$ and $\Delta>1.5 \mathrm{THz}$, respectively. Without RS and loss (not shown) the NF is $0 \mathrm{~dB}$ at $\Delta=0 \mathrm{THz}$ and increases below the respective curves with decreasing CE.

In conclusion, RS causes energy flow to or from the converted signal (the idler) depending on which configuration (i)-(iv) is used. Spontaneous RS unavoidably adds excess noise to the four-wave mixing process with a NF $22.2 \mathrm{~dB}$, which challenges the quantum state-preserving nature of BS. We have shown that to achieve the largest span of QFC with the smallest contribution from stimulated and spontaneous RS, distant BS, diagrams (iii)-(iv), is clearly preferable to nearby BS; specifically (iii) performing $3.25 \mathrm{THz} \mathrm{DC}$ with $\mathrm{CE} \approx 95 \%$ and $\mathrm{NF} \approx 2.5 \mathrm{~dB}$.

\section{References}

1. Raymer et al., Phys. Today 65, 32-37 (2012).

2. K. Inoue, IEEE Photon Technol. Lett. 6, 1451-1453 (1994).

3. Mechin et al., Opt. Express 14, 8995-8999 (2006).

4. McKinstrie et al., Phys. Rev. A 85, 053829 (2012).

5. Clark et al., Opt. Lett. 38, 947-949 (2013).

6. Friis et al., Opt. Express 21, 29320-29331 (2013).

7. Hollenbeck et al., J. Opt. Soc. Am. B 19, 2886-2892 (2002).

8. R. H. Stolen, Phys. Chem. Glasses 11, 83-87 (1970). 